\title{
Programmed cell death protein 1/programmed death ligand-1 checkpoint blockade meets patient-derived organoids
}

\author{
Lejia Sun", Huayu Yang", Yilei Mao \\ Department of Liver Surgery, Peking Union Medical College (PUMC) Hospital, PUMC \& Chinese Academy of Medical Sciences, Beijing 100730, \\ China \\ "These authors contributed equally to this work. \\ Correspondence to: Yilei Mao. Department of Liver Surgery Peking Union Medical College (PUMC) Hospital, PUMC \& Chinese Academy of \\ Medical Sciences 1\# Shuai-Fu-Yuan, Wang-Fu-Jing, Beijing 100730, China. Email: pumch-liver@hotmail.com.
}

Submitted Aug 20, 2019. Accepted for publication Oct 14, 2019.

doi: $10.21037 / \mathrm{atm} .2019 .11 .76$

View this article at: http://dx.doi.org/10.21037/atm.2019.11.76

Tumor immunotherapy, particularly programmed cell death protein 1 (PD-1)/programmed death ligand-1 (PD-L1) immune checkpoint blockade, has been instrumental in the successful treatment of cancer. Anti-PD-1/PD-L1 immunotherapy has been approved for many types of cancer $(1,2)$; however, it is generally understood that only a fraction of cancer patients benefit from the therapy, which limits the application of immune checkpoint inhibitors (3). Predicting the therapeutic responses to immune checkpoint inhibitors is a focus of many studies. Although many factors have been taken into consideration, such as clinicopathologic features, the level of PD-L1, tumor mutations, neoantigen load, and the status of microsatellite instability, the predicted performances of clinical parameters and biomarkers are not satisfactory $(4,5)$. New strategies for accurately predicting responses to immunotherapy are urgently needed. Patientderived organoids (PDOs) are a new method for predicting the response of PD-1/PD-L1 checkpoint blockade.

Tumor organoids are three-dimensional structures that are derived from tumor stem cells in vitro. PDOs can recapitulate many characteristics of the physiological structure and function of their matching primary tumor $(6,7)$. Furthermore, PDOs are similar to their parental tumors in phenotypic and genotypic profiling, and they share the same gene mutation spectrum (8). PDOs are an ideal alternative for cancer cell lines and patient-derived xenografts for predicting therapeutic responses and can also be used as human disease model for basic tumor immunity research (9). Previous studies suggest that PDOs are capable of predicting responses to chemotherapy and targeted drugs.
PDOs from patients with colorectal or gastroesophageal cancer had $100 \%$ accuracy in predicting a non-response to some drugs and $88 \%$ accuracy for predicting a positive drug response (10). However, it is more difficult to predict a therapeutic response to tumor immunotherapy in vitro because complicated immune factors are involved. Recently, Neal and colleagues developed tumor organoid modeling with the tumor immune microenvironment using air-liquid interface (ALI) cultures (11). The ALI PDOs were derived from 19 different tissue sites and included 28 unique tumor subtypes. The tumor architecture and integrated myofibroblast stroma were preserved in the ALI PDOs and CD8+ T cells, CD4+ T cells, B cells, natural killer, and natural killer $\mathrm{T}$ cells, macrophages were also retained. Moreover, ALI PDOs retained the conserved $T$ cell receptor repertoire of the parental tumor and displayed similar $\mathrm{T}$ cell clonal diversity. Importantly, PD-1/PD-L1 immune checkpoint blockade expanded and activated antigen-specific tumorinfiltrating lymphocytes (TILs) within ALI PDOs derived from melanoma, lung cancer, and renal cell carcinoma, and the results showed a similar clinical response rate as was observed in the ALI PDOs. ALI PDOs show potential as a tool to predict clinical responses to immune checkpoint therapies.

ALI PDOs are the first in vitro tumor model with an endogenous, syngeneic tumor microenvironment. The microenvironment is responsible for modifying tumor progression and therapeutic responses and it is preserved in ALI PDOs including the myofibroblast stromal elements and autologous immune cells. Three-dimensional organoid 
models with immune cells and a tumor microenvironment have been reported in the past; however, the tumor microenvironments were artificially reconstructed with heterogenous cells and did not contain multiple human immune cell types or functional T cells (12). Additionally, the TILs in these models exhibited responses to tumor immunotherapy without immune specificity (13).

ALI PDOs provide a new strategy for predicting responses to PD-1/PD-L1 immune checkpoint blockade. Reportedly, drug combinations prolong survival in patients compared with immune checkpoint inhibitors alone (14). Using ALI PDOs, it is easier to analyze effective drug combination regimens. However, the forecast accuracy of ALI PDOs needs to be further examined. Besides, ALI PDOs can also be used as bionic models for basic tumor immunity research.

Some drawbacks include that ALI PDOs have limited access to the peripheral immune system, which contributes to optimal anti-tumor immunity. Peripheral immune cells can be recruited to the tumor microenvironment and exert an influence on tumor immunotherapy (15). Second, there are no immunosuppressive cells, such as myeloid-derived suppressor cells (MDSCs), in ALI PDOs. Tumor immune escape is due to exhausted $\mathrm{T}$ cells as well as immunosuppressive cells and MDSCs are responsible for immune suppression and affect the efficacy of PD-1/ PD-L1 checkpoint blockade (16). Third, soluble immune factors, such as transforming growth factor (TGF)- $\beta$ and interleukin (IL)-10, have not been described in ALI PDOs. Therefore, it is necessary to carry out clinical tests to assess the sensitivity and specificity of predictions based on ALI PDOs.

Further improvements can be made to current ALI PDOs; for example, the addition of MDSCs and other essential components, such as endothelial cells. Furthermore, combining ALI PDOs and microfluidics would be helpful to evaluate drug response (17).

The uncertainty of anti-PD-1/PD-L1 immunotherapy has created the exigency for in vitro human prediction models that recapitulate the tumor immune microenvironment diversity, and ALI PDOs will enable the use of personalized tumor immunotherapy in the future.

\section{Acknowledgments}

Funding: This study was supported by grants from the CAMS Innovation Fund for Medical Sciences (CIFMS) (No. 2016-I2M-1-001) and the National High-tech Research and Development Projects (863) (No. 2015AA020303).

\section{Footnote}

Conflicts of Interest: The authors have no conflicts of interest to declare.

Ethical Statement: The authors are accountable for all aspects of the work in ensuring that questions related to the accuracy or integrity of any part of the work are appropriately investigated and resolved.

\section{References}

1. Abida W, Cheng ML, Armenia J, et al. Analysis of the Prevalence of Microsatellite Instability in Prostate Cancer and Response to Immune Checkpoint Blockade. JAMA Oncol 2019;5:471-8.

2. Gupta A, Budhu S, Merghoub T. One checkpoint may hide another: inhibiting the TGF $\beta$ signaling pathway enhances immune checkpoint blockade. Hepatobiliary Surg Nutr 2019;8:289-94.

3. Topalian SL, Drake CG, Pardoll DM. Immune checkpoint blockade: a common denominator approach to cancer therapy. Cancer Cell 2015;27:450-61.

4. Zhao P, Li L, Jiang X, et al. Mismatch repair deficiency/ microsatellite instability-high as a predictor for antiPD-1/PD-L1 immunotherapy efficacy. J Hematol Oncol 2019;12:54.

5. Takeda H, Nishikawa H, Osaki Y. The new era of precision medicine in hepatocellular carcinoma: the urgent need for promising biomarkers. Hepatobiliary Surg Nutr 2018;7:490-1.

6. Drost J, Clevers H. Organoids in cancer research. Nat Rev Cancer 2018;18:407-18.

7. Moro M, Casanova M, Roz L. Patient-derived xenografts, a multi-faceted in vivo model enlightening research on rare liver cancer biology. Hepatobiliary Surg Nutr 2017;6:344-6.

8. Aboulkheyr Es H, Montazeri L, Aref AR, et al. Personalized Cancer Medicine: An Organoid Approach. Trends Biotechnol 2018;36:358-71.

9. Yang H, Sun L, Liu M, et al. Patient-derived organoids: a promising model for personalized cancer treatment. Gastroenterol Rep (Oxf) 2018;6:243-5.

10. Vlachogiannis G, Hedayat S, Vatsiou A, et al. Patientderived organoids model treatment response of metastatic gastrointestinal cancers. Science 2018;359:920-6. 
11. Neal JT, Li X, Zhu J, et al. Organoid Modeling of the Tumor Immune Microenvironment. Cell 2018;175:197288.e16.

12. Finnberg NK, Gokare P, Lev A, et al. Application of 3D tumoroid systems to define immune and cytotoxic therapeutic responses based on tumoroid and tissue slice culture molecular signatures. Oncotarget 2017;8:66747-57.

13. Deng J, Wang ES, Jenkins RW, et al. CDK4/6 Inhibition Augments Antitumor Immunity by Enhancing T-cell Activation. Cancer Discov 2018;8:216-33.

14. Gong X, Qin S. Study progression of anti-angiogenetic therapy and its combination with other agents for

Cite this article as: Sun L, Yang H, Mao Y. Programmed cell death protein 1/programmed death ligand-1 checkpoint blockade meets patient-derived organoids. Ann Transl Med 2019;7(Suppl 8):S287. doi: 10.21037/atm.2019.11.76 the treatment of advanced hepatocellular carcinoma. Hepatobiliary Surg Nutr 2018;7:466-74.

15. Huang AC, Postow MA, Orlowski RJ, et al. T-cell invigoration to tumour burden ratio associated with antiPD-1 response. Nature 2017;545:60-5.

16. Grauers Wiktorin H, Nilsson MS, Kiffin R, et al. Histamine targets myeloid-derived suppressor cells and improves the anti-tumor efficacy of PD-1/PD-L1 checkpoint blockade. Cancer Immunol Immunother 2019;68:163-74.

17. Sontheimer-Phelps A, Hassell BA, Ingber DE. Modelling cancer in microfluidic human organs-on-chips. Nat Rev Cancer 2019;19:65-81. 